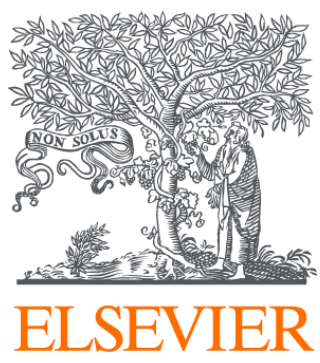

Since January 2020 Elsevier has created a COVID-19 resource centre with free information in English and Mandarin on the novel coronavirus COVID-

19. The COVID-19 resource centre is hosted on Elsevier Connect, the company's public news and information website.

Elsevier hereby grants permission to make all its COVID-19-related research that is available on the COVID-19 resource centre - including this research content - immediately available in PubMed Central and other publicly funded repositories, such as the WHO COVID database with rights for unrestricted research re-use and analyses in any form or by any means with acknowledgement of the original source. These permissions are granted for free by Elsevier for as long as the COVID-19 resource centre remains active. 


\title{
Change in age distribution of COVID-19 deaths with the introduction of COVID-19 vaccination
}

\author{
Roberta Pastorino $^{\mathrm{a}, 1}$, Angelo Maria Pezzullo ${ }^{\mathrm{b}, 1}$, Leonardo Villani ${ }^{\mathrm{b}, 1}$, \\ Francesco Andrea Causio ${ }^{\mathrm{b}}$, Cathrine Axfors ${ }^{\mathrm{c}}$, Despina G. Contopoulos-Ioannidis ${ }^{\mathrm{d}}$, \\ Stefania Boccia ${ }^{\mathrm{a}, \mathrm{b}}$, John P.A. Ioannidis ${ }^{\mathrm{c}, \mathrm{e}, *}$ \\ ${ }^{a}$ Department of Woman and Child Health and Public Health - Public Health Area, Fondazione Policlinico Universitario A. Gemelli IRCCS, Rome, Italy \\ ${ }^{\mathrm{b}}$ Section of Hygiene, University Department of Life Sciences and Public Health, Università Cattolica del Sacro Cuore, Rome, Italy \\ ${ }^{\mathrm{c}}$ Meta-Research Innovation Center at Stanford (METRICS), Stanford University, Stanford, CA, USA \\ ${ }^{\mathrm{d}}$ Division of Infectious Diseases, Department of Pediatrics, Stanford University School of Medicine, Stanford, CA, USA \\ ${ }^{\mathrm{e}}$ Department of Medicine, of Epidemiology and Population Health, of Biomedical Data Science, and of Statistics, Stanford University, Stanford, CA, USA
}

\section{A R T I C L E I N F O}

\section{Keywords:}

COVID-19

Death

Vaccination

Population data

\begin{abstract}
A B S T R A C T
Objectives: Most countries initially deployed COVID-19 vaccines preferentially in elderly populations. We aimed to evaluate whether population-level vaccine effectiveness is heralded by an increase in the relative proportion of deaths among non-elderly populations that were less covered by vaccination programs.

Eligible data: We collected data from 40 countries on age-stratified COVID-19 deaths during the vaccination period (1/14/2021-5/31/2021) and two control periods (entire pre-vaccination period and excluding the first wave).

Main outcome measures: We meta-analyzed the proportion of deaths in different age groups in vaccination versus control periods in (1) countries with low vaccination rates; (2) countries with age-independent vaccination policies; and (3) countries with standard age-dependent vaccination policies.

Results: Countries that prioritized vaccination among older people saw an increasing share of deaths among 0-69 year old people in the vaccination versus the two control periods (summary proportion ratio 1.32 [95 CI\% 1.24-1.41] and 1.35 [95 CI\% 1.26-1.44)]. No such change was seen on average in countries with ageindependent vaccination policies (1.05 [95 CI\% 0.78-1.41 and 0.97 [95 CI\% 0.95-1.00], respectively) and limited vaccination (0.93 [95 CI\% 0.85-1.01] and 0.95 [95 CI\% 0.87-1.03], respectively). Proportion ratios were associated with the difference of vaccination rates in elderly versus non-elderly people. No significant changes occurred in the share of deaths in age 0-49 among all 0-69 deaths in the vaccination versus pre-vaccination periods.

Conclusions: The substantial shift in the age distribution of COVID-19 deaths in countries that rapidly implemented vaccination predominantly among elderly provides evidence for the population level-effectiveness of COVID-19 vaccination and a favorable evolution of the pandemic towards endemicity with fewer elderly deaths.
\end{abstract}

\section{Introduction}

Several COVID-19 vaccines have shown very high efficacy for symptomatic infection in clinical trials (Polack et al., 2020; Voysey et al., 2021; Baden et al., 2021) and have also demonstrated high effectiveness for preventing hospitalization and death in preliminary analyses after wide deployment in large populations (Dagan et al., 2021; Vasileiou et al., 2021; Bernal et al., 2021). While they are diminishing the total mortality footprint from the epidemic waves, vaccines may also be expected to shift the demographics of COVID-19 fatalities, especially if the deployment of vaccines happens at different pace and intensity in different demographic groups.

\footnotetext{
* Corresponding author. Meta-Research Innovation Center at Stanford, Stanford Prevention Research Center, 1265 Welch Rd, MSOB X306, Stanford, CA, 94025, USA.

E-mail address: jioannid@stanford.edu (J.P.A. Ioannidis).

1 equal contribution.
} 
The aim of the study was to assess whether the proportion of deaths accounted for by non-elderly has increased in the mid-January through late May 2021 time period as compared with the previous phase of the pandemic in countries that have proceeded with vaccine roll-out in 2021 and that have detailed information of the age distribution of fatalities in different periods. The rationale was that almost all countries had a vaccination roll-out policy that started with the elderly and nursing homes (European Centre for Disea, 2021; Centers for Disease Contr, 2020). In most countries, very few young and middle age people were vaccinated in the first three months of roll out. Therefore, in the large majority of countries that used vaccines, the rates of vaccination were much higher in elderly versus non-elderly in early 2021 . However, the exact difference in vaccination rates between these two age strata varied a lot, mostly because of different rapidity in deploying vaccines. If vaccines are very effective for preventing deaths, one expects that the proportion of COVID-19 deaths accounted by elderly has decreased (and, conversely, the proportion of COVID-19 deaths accounted by non-elderly has increased) in the first 5 months of 2021, and that the change may depend on the differential rate of vaccination between elderly and non-elderly strata. Based on the same rationale, it was expected that countries that had no substantial roll-out of vaccines in the same period in any age group and those that aimed to vaccinate from the very early days all vulnerable people regardless of age would not experience a shift in the age distribution of COVID-19 deaths ceteris paribus. Evaluation of demographic change patterns would therefore offer an indirect way of assessing the effectiveness of vaccination for the hard outcome of death at the population level. We set out to assess these patterns using data from 40 countries with $>500$ COVID- 19 deaths by end of May 2021. We also try to discuss our findings in the context of and against other factors (besides deployment of vaccination) that might also affect whether the age distribution of COVID-19 deaths changes over time.

\section{Methods}

\subsection{Data on COVID-19 deaths and definitions of contrasted time periods}

We considered all countries that had a total of COVID-19 deaths $>500$ as of end of May 2021, and which had publicly-available agestratified information on COVID-19 deaths so as to separate elderly versus non-elderly age strata with a cut-off at 70 years (or 65 or 60, if 70 years cut-off data were unavailable). The selected cut-off is similar to what was used in a previous analysis of age distribution in the first versus second wave of the pandemic until January 2021 (Institut national d'études démographiques (INED), 2020).

We collected data on deaths from Institut national d'études démographiques (INED) (Institut national d'études démographiques (INED), 2020), COVerAGE-DataBase (Riffe et al., 2021), and other national databases (National Institute of Population and Social Security Research - Japan, 2021; Serwis Rzeczypospolitej P; National Health Institute, 2021; Fornos, 2021). England and Wales were grouped because their mortality data were reported together. All the sources about deaths are shown in supplementary table S1. Of 122 countries with $>500$ COVID-19 deaths (Worldometer) eligible age-stratified data were eventually available for 40 countries.

We calculated the number of deaths from January 14, 2021 (or as close as possible if data on this date were unavailable) until the latest date with data available at the time of our searches (between the end of May and the beginning of June 2021); this is called the "vaccination period". January 14, 2021 was selected, because, while vaccinations started in December 2020 in most of the analyzed countries, any meaningful benefit is unlikely to have been seen at the population level by early January. For comparison, we used two control periods preceding the vaccination period: the "entire pre-vaccination period", i.e. from the onset of the pandemic; and the "pre-vaccination second wave period" which only considered deaths during the second wave of the pandemic up until the start of the vaccination period. Deaths during the latter control period offer a more proximal comparison to vaccination period and they were calculated by excluding deaths that had occurred in the first wave. The first wave period was defined as in a previous analysis (Ioannidis et al., 2021) and the data on deaths during the first wave were taken directly from that analysis and updated if more recently data were available. For the countries that had not been included in that previous analysis, we used for consistency as the separating date between the first and second waves the date with trough (lowest number) of deaths for a 7-day average according to Worldometer (Worldometer). When data were not available specifically up to the trough date, we considered a separation date for the two waves that was as close as possible to the trough. Information about the trough and the separation dates are shown in table S1.

Age-stratified death counts for the time periods of interest were separately recorded for three age groups: $0-49,50-69$, and $\geq 70$ years. Data were instead available for the age groups $0-44,45-64$, $\geq 65$ for Austria, Belgium, and USA; 0-39, 40-59, $\geq 60$ for Bangladesh and Northern Ireland; and 0-44, 45-59 and $\geq 60$ for Indonesia. Deaths in chronic care establishments and in other social or medico-social residential facilities in France were counted in the $\geq 70$ age group.

There were no data available on deaths that occurred in the second wave pre-vaccination period with suitable age stratification for Canada, Israel, Jamaica, Jordan, Northern Ireland and Uruguay.

\subsection{Vaccination data}

We obtained data on COVID-19 vaccination coverage (at least one dose received and full vaccination) from international databases for the general population and for the $0-69$ and $\geq 70$ year age groups. Number of vaccinations in the age group was extracted from the cited databases, and if the corresponding population denominator was not provided by the source, we derived it from publicly available population pyramids (PopulationPyramid.net. Po, 1950). Sources and vaccination coverage by age groups $0-69, \geq 70$ and overall are available in the supplementary table S2. In order to consider the effect of COVID-19 vaccination on deaths, we used the vaccination coverage until April 30, 2021 (or as close as possible if data on this date were unavailable). This date was chosen to allow a 1-month difference between the vaccination data and the end of the period during which deaths were counted.

\subsection{Contrasts of age groups and data synthesis}

The main analysis compared the proportion of COVID-19 deaths in the age group 0-69 (0-59 or 0-64 when these were not available)/per total COVID-19 deaths in the vaccination period versus each of the two pre-vaccination control periods. Proportion ratios for each country (proportion of COVID-19 deaths accounted by 0-69 year old people in the vaccination period divided by the proportion of COVID-19 deaths accounted by the same age stratum in a control period for the same country) were calculated along with $95 \%$ confidence intervals and were synthesized across countries with inverse-variance random effects metaanalysis. Between-country heterogeneity was measured using the $\mathrm{I}^{2}$ statistic and tested with the Q test. The meta-analysis was also stratified in three categories: 1) countries with low vaccination rates, i.e. proportion of vaccination with at least one dose $<5 \%$ in the general population by late April 2021 (Japan, Republic of Moldova, Peru, Ukraine, Philippines, Bangladesh, Jamaica, and Indonesia); 2) countries with ageindependent policies, i.e. adopting very early (before $1 / 15 / 2021$ ) vaccination policies for vulnerable groups regardless of age (Romania, Denmark, Israel) (University of Oxford - Bl, 2020); 3) countries with standard age-dependent policies (Argentina, Austria, Belgium, Brazil, Canada, Colombia, England and Wales, Finland, France, Germany, Greece, Hungary, Italy, Jordan, Mexico, Netherlands, Northern Ireland, Norway, Poland, Portugal, Scotland, Slovenia, South Korea, Spain, Sweden, Switzerland, Uruguay, and USA). As discussed above, a change 
Table 1

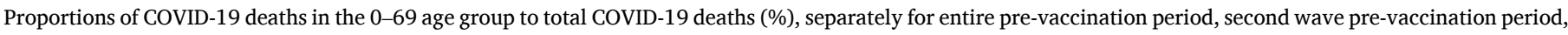
and vaccination period.

\begin{tabular}{|c|c|c|c|}
\hline Country & Entire pre-vaccination period & Second wave pre-vaccination period & Vaccination period \\
\hline Argentina & $17,597 / 47,665$ (36.9) & $8877 / 23,524(37.7)$ & $14,732 / 31,625$ (46.6) \\
\hline Australia & $58 / 909(6.4)$ & $44 / 812(5.4)$ & $0 / 1(0.0)$ \\
\hline Austria & $422 / 6828(6.2)$ & $384 / 6183(6.2)$ & $374 / 3485(10.7)$ \\
\hline Bangladesh & $4781 / 7835$ (61.0) & $4309 / 7086(60.8)$ & $2172 / 3560(61.0)$ \\
\hline Belgium & $1232 / 20,224(6.1)$ & $694 / 10,613(6.5)$ & $442 / 4418(10.0)$ \\
\hline Brazil & $94,070 / 212,007(44.4)$ & $21,887 / 50,274(43.5)$ & $151,324 / 256,026(59.1)$ \\
\hline Canada $^{a}$ & $1883 / 17,315(10.9)$ & - & $1593 / 7692(20.7)$ \\
\hline Colombia & $24,207 / 55,034(44.0)$ & $9570 / 23,420(40.9)$ & $24,432 / 47,669(51.3)$ \\
\hline Denmark & $203 / 1720(11.8)$ & $128 / 1122(11.4)$ & $88 / 791(11.1)$ \\
\hline England and Wales & $15,104 / 94,132(16.0)$ & $6722 / 42,493(15.8)$ & $9037 / 44,033(20.5)$ \\
\hline Finland & $67 / 594(11.3)$ & $33 / 286(11.5)$ & $72 / 390(18.5)$ \\
\hline France & $7397 / 69,044(10.7)$ & $3702 / 38,816(9.5)$ & $5684 / 39,706(14.3)$ \\
\hline Germany & $4629 / 41,486(11.2)$ & $3303 / 32,343(10.2)$ & $6734 / 45,749(14.7)$ \\
\hline Greece & $835 / 5421(15.4)$ & $793 / 5246(15.1)$ & $1091 / 6495(16.8)$ \\
\hline Hungary & $3047 / 11,066(27.5)$ & $2918 / 10,477$ (27.9) & $6635 / 18,708(35.5)$ \\
\hline Indonesia & $14,137 / 25,245$ (56.0) & $10,382 / 19,173$ (54.1) & $11,819 / 26,199$ (45.1) \\
\hline Israel $^{\mathrm{a}}$ & $770 / 3704(20.8)$ & - & $728 / 2630(27.7)$ \\
\hline Italy & $10,974 / 78,591(14.0)$ & $5759 / 42,947$ (13.4) & $7178 / 44,684(16.1)$ \\
\hline Jamaica $^{a}$ & $141 / 312(45.2)$ & - & $312 / 638(48.9)$ \\
\hline Japan & $421 / 3338(12.6)$ & $294 / 2512(11.7)$ & $657 / 6428(10.2)$ \\
\hline Jordan $^{\mathrm{a}}$ & $1406 / 4040(34.8)$ & - & $2097 / 5469$ (38.3) \\
\hline Mexico & $102,687 / 156,933(65.4)$ & $39,175 / 62,379$ (62.8) & $37,297 / 60,412$ (61.7) \\
\hline Netherlands & $1215 / 12,563(9.7)$ & $520 / 6428(8.1)$ & $658 / 5003(13.2)$ \\
\hline Northern Ireland ${ }^{\mathrm{a}}$ & $76 / 1532(5.0)$ & - & $39 / 621(6.3)$ \\
\hline Norway & $76 / 511(14.9)$ & $47 / 276(17.0)$ & $71 / 272(26.1)$ \\
\hline Peru & $52,794 / 96,610(54.6)$ & $3233 / 6477$ (49.9) & $50,240 / 87,885(57.2)$ \\
\hline Philippines & $7724 / 11,509(67.1)$ & $6864 / 10,214(67.2)$ & $5625 / 8731(64.4)$ \\
\hline Poland & $8036 / 26,371(30.5)$ & $7575 / 24,775(30.6)$ & $12,664 / 39,260(32.3)$ \\
\hline Portugal & $1050 / 8543(12.3)$ & $811 / 6793$ (11.9) & $1158 / 8479$ (13.7) \\
\hline Republic of Moldova & $1743 / 2942(59.2)$ & $1190 / 2058$ (57.8) & $1418 / 2663(53.2)$ \\
\hline Romania & $7251 / 17,021(42.6)$ & $6268 / 15,739(39.8)$ & $5077 / 13,096(38.8)$ \\
\hline Scotland & $727 / 7467$ (9.7) & $346 / 3237$ (10.7) & $405 / 2647$ (15.3) \\
\hline Slovenia & $177 / 3423(5.2)$ & $172 / 3313(5.2)$ & $112 / 1270(8.8)$ \\
\hline South Korea & $189 / 1195$ (15.8) & $138 / 970(14.2)$ & $132 / 745(17.7)$ \\
\hline Spain & $7831 / 57,080(13.7)$ & $3638 / 27,376(13.3)$ & $3800 / 22,417$ (17.0) \\
\hline Sweden & $878 / 9940(8.8)$ & $291 / 4248(6.9)$ & $510 / 3642(14.0)$ \\
\hline Switzerland & $638 / 7904(8.1)$ & $473 / 6264(7.6)$ & $285 / 2346(12.1)$ \\
\hline Ukraine & $10,382 / 20,305(51.1)$ & $9916 / 19,524(50.8)$ & $12,230 / 26,849(45.6)$ \\
\hline Uruguay $^{\mathrm{a}}$ & $77 / 280(27.5)$ & - & $1353 / 3755(36.0)$ \\
\hline USA & $79,806 / 416,640(19.2)$ & $53,136 / 285,179(18.6)$ & $37,153 / 162,388$ (22.9) \\
\hline
\end{tabular}

${ }^{\text {a }}$ Deaths that occurred in the second wave were not available in suitable age stratification.

in the age demographics of deaths was expected to have occurred only in the third group. Since Australia registered only one death in the vaccination period it was excluded from analyses. Of note, even in countries without age restrictions, older people may still be more motivated than younger ones to be vaccinated faster. Therefore, intended policy is only a surrogate of what eventually happened in terms of vaccination rates across different age groups and some within-group heterogeneity may be unavoidable.

A secondary analysis compared the proportion of COVID-19 deaths in the age group $0-49$ years $(0-39$ or $0-44$ when these were not available) among all COVID-19 deaths in the age group 0-69 (0-59 or 0-64 when these were not available) in the vaccination period versus each of the two pre-vaccination control periods. Vaccination rates were limited in people $<70$ years old by April 2021 and therefore, no change in the age demographics was expected to have occurred when these periods were compared. Meta-analysis of proportion ratios across countries used random effects similar to the primary analysis.

Finally, we performed exploratory meta-regression analyses, where we examined if the natural logarithm of the proportion ratio of deaths in the 0-69 age group in the vaccination versus each control period was associated with the difference in the proportion of people who were vaccinated by the end of April in those $\geq 70$ versus those $0-69$ years old (using a 60 or 65 years cut-off, if data for the 70 years cut-off were not available). This difference shows the strength of the contrast in vaccination rates between the two age groups of interest and we hypothesized that it may explain in part the differential change in deaths in the two age strata. The meta-regression analysis was weighted by the inverse of the variance of the natural logarithm of the proportion ratio. We considered two different versions of the meta-regression, one using the data on the proportion of people who were fully vaccinated and another using the data on the proportion of people who had received at least one dose, given that vaccine efficacy may be substantial even within 1-2 weeks after a single dose (Vasileiou et al., 2021; Bernal et al., 2021). For the countries of Group 1 (those with low vaccination rates) we had vaccinations per age strata only for Japan and Peru, while for the countries of Group 3 we had missing data for six countries. We imputed the missing values in each group as the median of the differences in the proportion of vaccinated people between the relevant age strata of the countries with observed data in their group. The countries of Group 2 that very early vaccinated all vulnerable groups regardless of age (Romania, Denmark, and Israel) were excluded from this analysis.

\subsection{Statistical analyses}

All analyses were performed in STATA 16.0 (StataCorp, 2019). We used $\mathrm{P}<0.005$ as the threshold for statistical significance and values between 0.005 and 0.05 were considered suggestive (Benjamin et al., 2018). 


\section{Results}

\subsection{Country characteristics}

Of the 40 included countries, the majority $(n=27)$ were highincome, 9 were upper-middle income, and 4 were lower-middle income; the majority $(n=23)$ were in Europe, 9 in America, 7 in Asia, and 1 in Oceania; and the majority $(n=31)$ had a median age of the population exceeding 31 years. The 8 countries in group 1 (those with low vaccination rates) differed from the others in having lower income (4 were lower-middle, 3 was upper-middle, and 1 was high-income) and being less likely to be in Europe (only 2/8) and to have a median age of $>32$ in their population (only $3 / 8$ ).

\subsection{Deaths and vaccination rates per age strata}

Table 1 reports the number of COVID-19 deaths in people 0-69 years old and the total COVID-19 deaths in each of the 40 eligible countries with data for the two control periods, and the vaccination period. The proportion of COVID-19 deaths that had occurred in people 0-69 years old ranged from $5.0 \%$ to $67.1 \%$ in the entire pre-vaccination period, from $5.2 \%$ to $67.2 \%$ in the second wave pre-vaccination period, and from $0.0 \%$ to $64.4 \%$ in the vaccination period (median $15.6 \%, 14.7 \%$, and $20.6 \%$, respectively). The proportions were much lower in highincome countries (range 5.0-30.5\% [median 11.8\%], 5.2-30.6\% [median $11.5 \%$ ], and $0.0 \%-36.0 \%$ [median $15.3 \%$ ], in the three periods, respectively).

Table 2 reports the data about vaccination coverage in each of the 40 countries by the end of April in the 0-69 and $\geq 70$ years old age strata and in the overall population. The proportion of the general population that had been fully vaccinated varied from $0.0 \%$ to $57.4 \%$ across the 40 countries, while for those who had received at least one dose the proportion varied from $1.5 \%$ to $61.4 \%$ across the 40 countries. The proportion of the population 0-69 years old that had been fully vaccinated ranged from $1.3 \%$ to $53.8 \%$, and $1.5 \%-57.9 \%$ had received at least one dose. Conversely, the proportion of the population $\geq 70$ years old with full vaccination ranged from $0.0 \%$ to $99.7 \%$, and $0.6 \%-100.0 \%$ had received at least one dose.

\subsection{Change in age distribution over time}

As shown in Fig. 1, the proportion of deaths accounted for by those 0-69 years old increased in the vaccination period compared with the entire pre-vaccination period (panel A) or the second wave prevaccination period (panel B) with summary proportion ratios being 1.20 (95\% CI, 1.14-1.27) and 1.22 (95\% CI, 1.15-1.30), respectively (p

Table 2

COVID-19 vaccination coverage by age groups and overall (\%).

\begin{tabular}{|c|c|c|c|c|c|c|c|}
\hline \multirow[t]{2}{*}{ Country } & \multicolumn{2}{|l|}{$0-69$} & \multicolumn{2}{|l|}{$\geq 70$} & \multicolumn{2}{|l|}{ Overall } & \multirow[t]{2}{*}{ Date } \\
\hline & At least one dose & fully vaccinated & At least one dose & fully vaccinated & At least one dose & fully vaccinated & \\
\hline Argentina & 10.3 & 1.8 & $100.0^{c}$ & 7.9 & 15.9 & 2.2 & Apr 30, 2021 \\
\hline Australia & NA & NA & NA & NA & NA & NA & Apr 30, 2021 \\
\hline Austria & 20.0 & 5.9 & 69.8 & 35.7 & 23.5 & 9.0 & Apr 25, 2021 \\
\hline Bangladesh & NA & NA & NA & NA & 3.5 & 1.7 & Apr 29, 2021 \\
\hline Belgium & 13.9 & 5.0 & 86.4 & 17.6 & 23.9 & 6.7 & Apr 25, 2021 \\
\hline Brazil & 8.9 & 3.2 & 98.3 & 69.3 & 14.1 & 7.0 & Apr 30, 2021 \\
\hline Canada & 21.2 & 1.6 & 85.6 & 8.7 & 29.3 & 2.5 & Apr 24, 2021 \\
\hline Colombia & NA & NA & NA & NA & 6.6 & 3.0 & Apr 30, 2021 \\
\hline Denmark & 9.6 & 4.9 & 89.4 & 40.5 & 21.1 & 10.0 & Apr 25, 2021 \\
\hline England & 42.6 & 9.8 & $100.0^{c}$ & 82.3 & 50.0 & 19.5 & Apr 25, 2021 \\
\hline Finland & 16.0 & 2.1 & 91.6 & 6.7 & 28.0 & 2.9 & Apr 25, 2021 \\
\hline France & 12.7 & 2.9 & 70.6 & 39.2 & 21.2 & 8.2 & Apr 25, 2021 \\
\hline Germany & NA & NA & NA & NA & 23.7 & 7.2 & Apr 25, 2021 \\
\hline Greece & 12.6 & 3.2 & 61.9 & 33.3 & 18.7 & 7.6 & Apr 25, 2021 \\
\hline Hungary & 31.0 & 11.4 & 75.4 & 53.8 & 36.9 & 17.0 & Apr 25, 2021 \\
\hline Indonesia & NA & NA & NA & NA & 4.3 & 2,5 & Apr 25, 2021 \\
\hline Israel & 57.9 & 53.8 & $100.0^{c}$ & 99.7 & 61.4 & 57.4 & Apr 30, 2021 \\
\hline Italy & 10.7 & 4.5 & 64.7 & 28.5 & 20.9 & 8.7 & Apr 25, 2021 \\
\hline Jamaica & NA & NA & NA & NA & 4.9 & 0.2 & May 14, 2021 \\
\hline Japan $^{\mathrm{b}}$ & 2.1 & 1.5 & 0.6 & 0.0 & 1.8 & 1.2 & Apr 30, 2021 \\
\hline Jordan & NA & NA & NA & NA & 6.9 & 2.2 & Apr 29, 2021 \\
\hline Mexico $^{\mathrm{a}}$ & 1.5 & 1.3 & 73.6 & 38.4 & 9.6 & 5.5 & Apr 30, 2021 \\
\hline Netherlands & NA & NA & NA & NA & 25.6 & 6.9 & Apr 25, 2021 \\
\hline Northern Ireland & NA & NA & NA & NA & 49.8 & 22.3 & Apr 30, 2021 \\
\hline Norway & 19.9 & 6.5 & 87.7 & 33.6 & 22.2 & 5.6 & Apr 25, 2021 \\
\hline Peru & 2.2 & 1.8 & 19.9 & 2.8 & 3.2 & 1.9 & Apr 30, 2021 \\
\hline Philippines & NA & NA & NA & NA & 1.5 & 0.3 & Apr 30, 2021 \\
\hline Poland & 14.9 & 2.9 & 62.6 & 36.1 & 20.5 & 6.8 & Apr 25, 2021 \\
\hline Portugal & 11.0 & 3.0 & 77.4 & 34.4 & 21.7 & 8.1 & Apr 25, 2021 \\
\hline Republic of Moldova & NA & NA & NA & NA & 3.2 & 0.4 & Apr 30, 2021 \\
\hline Romania $^{\mathrm{a}}$ & 10.8 & 5.8 & 24.6 & 16.6 & 15.8 & 9.3 & Apr 25, 2021 \\
\hline Scotland & 43.6 & 13.2 & $100.0^{c}$ & 89.2 & 51.3 & 23.6 & Apr 30, 2021 \\
\hline Slovenia & 12.9 & 2.3 & 61.5 & 46.5 & 19.6 & 8.4 & Apr 25, 2021 \\
\hline South Korea & NA & NA & NA & NA & 6.5 & 0.5 & Apr 30, 2021 \\
\hline Spain & 38.3 & 14.5 & 79.5 & 34.4 & 47.1 & 26.2 & Apr 25, 2021 \\
\hline Sweden & 31.2 & 12.4 & 90.0 & 33.0 & 40.5 & 21.7 & Apr 25, 2021 \\
\hline Switzerland & 9.9 & 4.1 & 69.2 & 45.6 & 18.4 & 10.1 & Apr 25, 2021 \\
\hline Ukraine & NA & NA & NA & NA & 1.7 & 0.0 & Apr 30, 2021 \\
\hline Uruguay $^{\mathrm{b}}$ & 27.3 & 18.0 & 71.6 & 30.1 & 34.0 & 19.8 & Apr 30, 2021 \\
\hline $\mathrm{USA}^{\mathrm{b}}$ & 35.1 & 22.4 & 82.6 & 69.1 & 42.9 & 30.2 & Apr 30, 2021 \\
\hline
\end{tabular}

NA: Data were not available.

${ }^{a}$ Age group 0-59 and $\geq 60$.

b Age group 0-64 and $\geq 65$.

c More vaccinated people than the estimated population in this age category. 

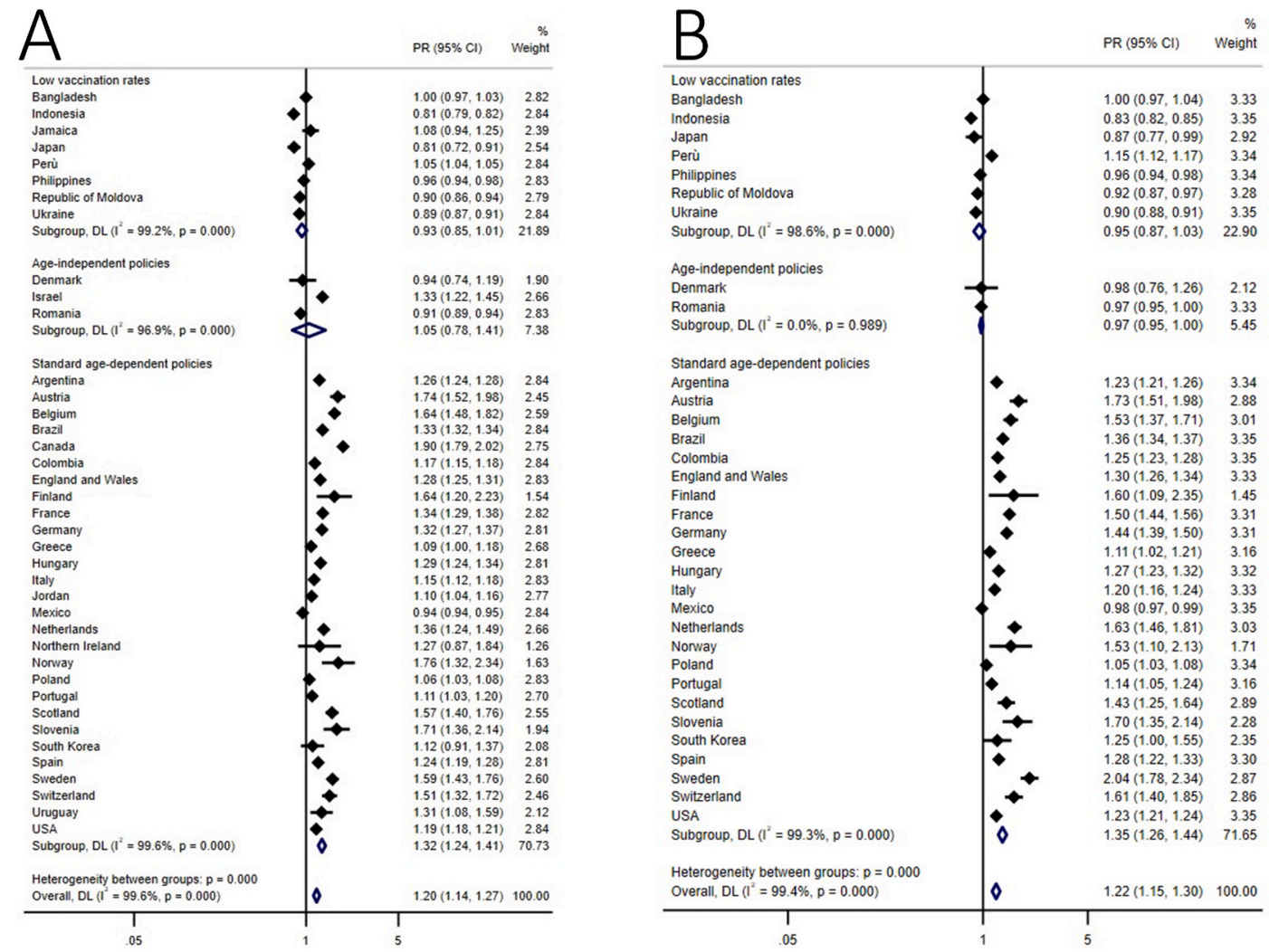

Fig. 1. Meta-analysis of proportion ratios (PR) of COVID-19 deaths in age 0-69 in the vaccination versus pre-vaccination control periods stratified according to three main categories 1) Low vaccination rates: countries with a proportion of vaccination with at least one dose $<5 \%$ in the general population by the end of April 2021; 2) Age-independent policies: countries with early vaccination policies that vaccinated vulnerable groups regardless of age on January 15, 2021; 3) Standard agedependent policies: remaining countries. PR $>1$ implies a higher proportion of deaths accounted by 0-69 age group in the vaccination period versus prevaccination periods. Panel A: vaccination period versus entire pre-vaccination period. Panel B: vaccination period versus second wave pre-vaccination period.

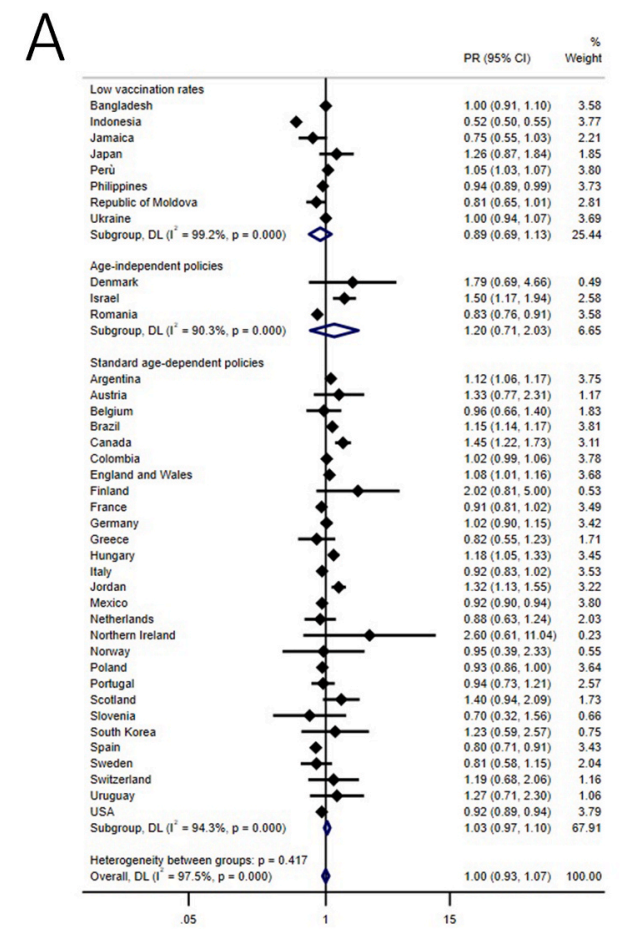

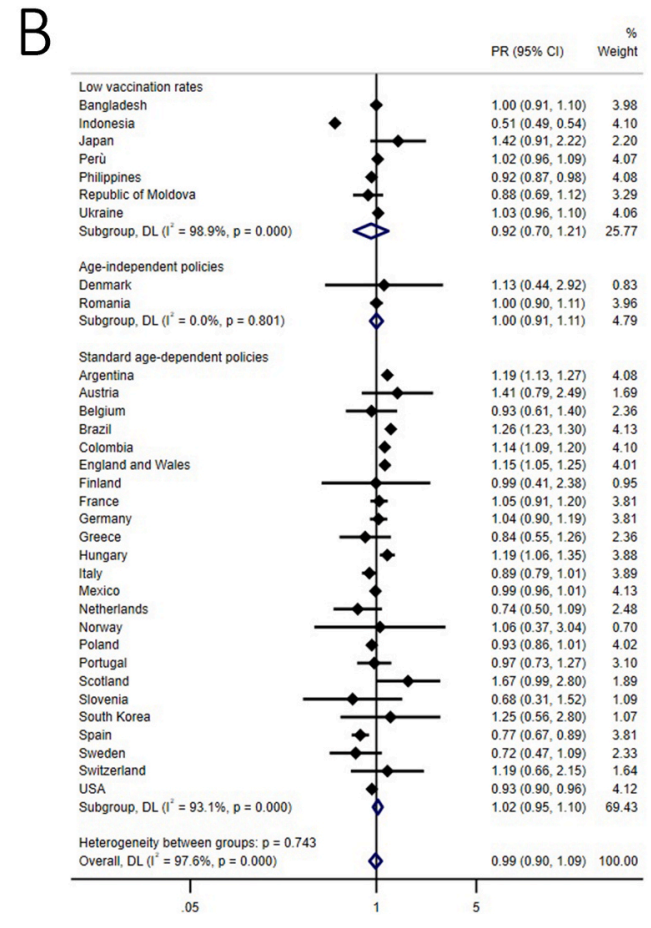

Fig. 2. Meta-analysis of proportion ratios (PR) of COVID-19 deaths in age $0-49$ among 0-69 deaths in the vaccination versus prevaccination control periods stratified by three main categories 1) Low vaccination rates: countries with a proportion of vaccination with at least one dose $<5 \%$ in the general population by the end of April 2021; 2) Age-independent policies: countries with early vaccination policies that vaccinated vulnerable groups regardless of age on January 15, 2021; 3) Standard age-dependent policies: remaining countries. PR $>1$ implies a higher proportion of deaths accounted by 0-49 age group in the vaccination period versus pre-vaccination periods. Panel A: vaccination period versus entire prevaccination period. Panel B: vaccination period versus second wave pre-vaccination period. 
$<0.0001$ for both) when all countries were considered, but there was extreme between-country heterogeneity. An increase of the proportion of deaths accounted for by the non-elderly was seen in all countries with substantial vaccination and not early age-inclusive policies (except for Mexico) with summary proportion ratios of 1.32 (95\% CI, 1.24-1.41) and 1.35 (95\% CI, 1.26-1.44), respectively, but there was still large between-country heterogeneity in the exact magnitude of the proportion ratio. For the countries of Group 1 (Japan, Republic of Moldova, Peru, Ukraine, Philippines, Bangladesh, Jamaica, and Indonesia) where very little vaccination happened in any age group, there was no difference in the proportion of deaths accounted for by 0-69 years old people on average (summary proportion ratio 0.93 (95\% CI, 0.85-1.01) and 0.95 (95\% CI, 0.87-1.03), respectively). Same pattern of no overall age shift was documented for the countries of Group 2 (Denmark, Romania and Israel) (summary proportion ratio 1.05 (95\% CI, 0.78-1.41) and 0.97 (95\% CI, 0.95-1.00), respectively). Between-country heterogeneity existed also in the results of countries from these two groups.

As shown in Fig. 2, there was no significant, consistent pattern of change in the age distribution of deaths in the $0-49$ age strata in the vaccination period versus the pre-vaccination control periods (summary proportion ratio 1.00 (95\% CI 0.93-1.07) and 0.99 (95\% CI 0.90-1.09), $\mathrm{p}>0.05$ for both).

\subsection{Regression analyses}

The regression analyses provided a suggestive relationship for the proportion ratio of deaths in the non-elderly in the vaccination versus control pre-vaccination periods and the difference of the proportion of fully vaccinated people in the $\geq 70$ versus the $0-69$ years group ( $\mathrm{p}=$
0.018 and $\mathrm{p}=0.009$ ) (Fig. 3). The difference of the proportions of people vaccinated with at least one dose between the two age strata had a significant association with the proportion ratio of deaths in the nonelderly ( $\mathrm{p}<0.0001$ and $\mathrm{p}<0.0001$ ) (Fig. 3).

\section{Discussion}

\subsection{Main findings}

Data from 40 countries show that the use of anti-SARS-CoV-2 vaccination was associated with a marked change in the age distribution of COVID-19 deaths in the first 5 months of 2021 in countries that prioritized vaccination among older people, with a relative increase in the share of deaths among non-elderly people. Conversely, on average, there was no change in the age distribution of COVID-19 deaths during the same time period in countries adopting very early vaccination policies that aimed to vaccinate all the vulnerable people regardless of age and in countries that had very limited use of vaccination.

For countries that prioritized vaccination among elderly people, the increase in the proportion of deaths among the non-elderly was about 1.35 -fold. Conversely, there was no change in the relative proportion of COVID-19 deaths among those 0-49 versus those 50-69 years old, which is consistent with the fact that vaccination rates in these two groups did not differ markedly in the first 4 months of 2021 in the vast majority of countries. Exploratory regression suggested that the age shift with a larger share of deaths among the non-elderly was associated with how stark the difference in vaccination rates had been in the elderly versus non-elderly strata. The association was seen most clearly for the difference in the proportion of receiving at least one vaccine dose, with less
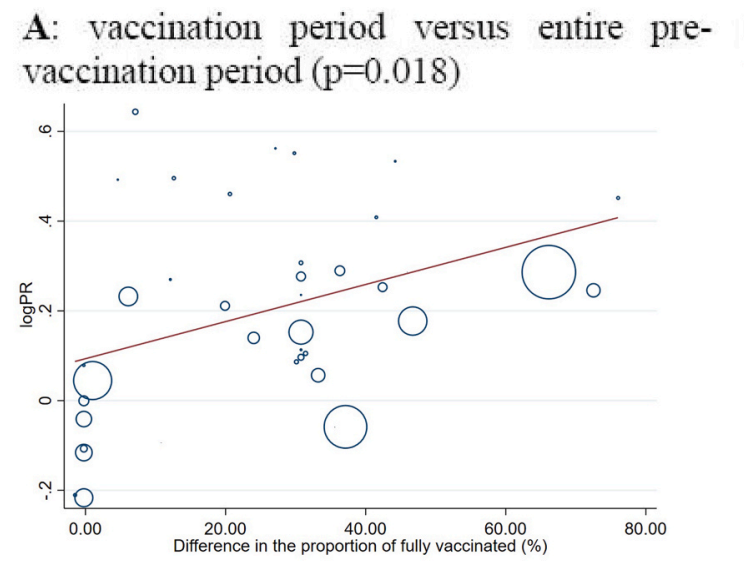

C: vaccination period versus entire prevaccination period. $(p<0.0001)$

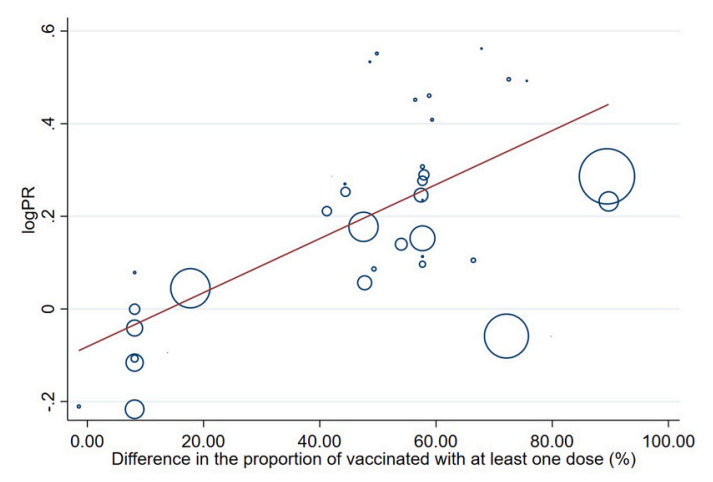

B: vaccination period versus second wave prevaccination period $(p=0.009)$

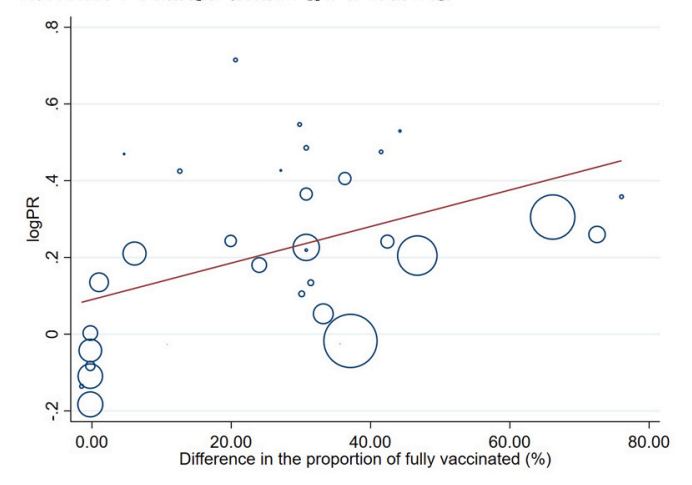

D: vaccination period versus second wave prevaccination period ( $\mathrm{p}<0.0001$ )

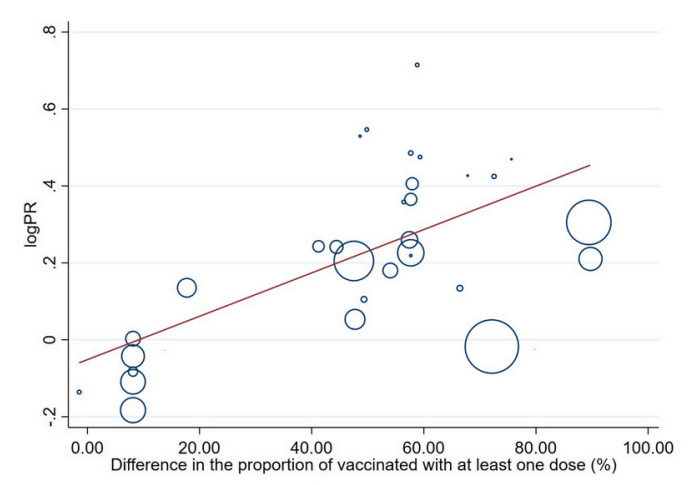

Fig. 3. Meta-regression of the logarithm of the proportion ratio of COVID-19 deaths in the 0-69 age group in the vaccination versus pre-vaccination periods as a function of the difference in the proportion of fully vaccinated people in the $\geq 70$ versus $0-69$ age strata (Panel A and B) and as a function of the difference in the proportion of vaccinated people with at least one dose in the $\geq 70$ versus $0-69$ age strata (Panel C and D). 
clear association when data on the rates of full vaccination were examined. This may reflect the fact that a single dose of the widely used vaccines already confers within 2 weeks substantial protection. Moreover, the proportion of people fully vaccinated was much smaller, even in the most elderly strata, probably making the association more difficult to document.

Countries with low vaccination rates were often (not always) nonEuropean, non-high-income countries with young population age pyramids. Not only vaccination, but also most non-vaccine public health and other resources may be limited in many such countries and thus the ability to make a difference in the course of the epidemic and the agestructure of victims thereof may be more limited in general. The impact of vaccination would be clearer in contrasts of countries in the same region that had similar income and age-structure. Indeed, such a contrast is offered for high-income countries by Japan (low early vaccination) versus South Korea (more substantial early vaccination) where the proportion ratios of deaths in age 0-69 years were indeed opposite ( 0.87 in Japan versus 1.25 in South Korea) in the vaccination versus second wave pre-vaccination period; and to a lesser extent for middle-income countries by Peru versus Colombia (1.15 in Peru versus 1.25 in Colombia, respectively, for the same contrast).

\subsection{Inferences from the observed age shift}

These data offer indirect, but strong support for the ability of vaccination programs to decrease COVID-19 mortality as they can change substantially the death demographics. Apparently, vaccination markedly reduced the population fatality burden among the elderly, the age group carrying the lion's share of the mortality burden since the beginning of the pandemic. However, it should be noted that despite the substantial age shift in fatalities, the elderly continued to represent the vast majority of deaths in most high-income countries despite the rapid vaccination of a large share of this group. The residual death burden may reflect the fact that many elderly were still not vaccinated in these countries during the period of interest; furthermore, some vulnerable elderly may have launched inefficient immune responses to vaccination (Connors et al., 2021; Hussien et al., 2021; Koff et al., 2021). Hopefully, the former reason may be the more common one, as clinical trials suggest very good immunogenicity in the majority of the elderly for several COVID-19 vaccines. Thorough vaccination of the elderly may dramatically decrease the total footprint of COVID-19 fatalities.

There is no reason to believe that COVID-19 vaccination may not be equally effective (if not more effective) in curtailing fatalities at the population level among non-elderly individuals. During the pandemic, the non-elderly have accounted for a small portion of deaths in highincome countries. Conversely, among the countries that we evaluated, deaths at age $<70$ have been the majority of documented COVID-19 deaths during the pandemic in Bangladesh, Mexico, Peru, and Moldova, and they have represented $40-50 \%$ of the COVID-19 deaths in Brazil, Colombia, Indonesia, Jamaica, and Ukraine. Mortality data for these countries need to be seen with some caution, as documentation of COVID-19 may have been more incomplete. If anything, in several of these countries like Indonesia, Mexico, Philippines, Moldova and Ukraine, there was a suggestion that the proportion of elderly COVID-19 deaths increased in 2021 versus in 2020, despite no or little vaccination of the population. These changes may represent changes in the documentation of COVID-19 deaths, especially among the elderly, e.g. more deaths missed in 2020 due to insufficient testing (Ioannidis, 2021a) - or other data artefacts.

\subsection{Potential limitations}

Some other limitations need to be discussed. The results presented here represent exploratory analyses of ecological nature and they carry the risk of ecological fallacy and unmeasured, unaccounted confounding. Nevertheless, the patterns observed are compatible and extend the results of large-scale population cohort analyses from countries like Israel and UK that suggested large clinical benefits from early rapid massive deployment of vaccination in whole countries (Dagan et al., 2021; Vasileiou et al., 2021; Bernal et al., 2021). One particular source of unaccounted confounding is the extent to which COVID-19 deaths in nursing home residents may have decreased over time, due to non-vaccine-related reasons, e.g. better protection of nursing homes after the first wave in 2020 and a reduction due to harvesting effects of the population pool of frail nursing home residents who would be highly likely to succumb upon infection. A previous analysis comparing the first versus the second wave (until mid-January 2021) found a large decrease in the proportion of COVID-19 represented by nursing home residents in several high-income countries (Institut national d'études démographiques (INED), 2020). Available data from several European countries and Canada suggest a further decline in the share of deaths represented by nursing home residents during 2021 (Appendix 1). Universal vaccination is easier to achieve in nursing homes than among the community-dwelling elderly. Nevertheless, deaths seem to have declined equally steeply also among community-dwelling elderly (Appendix 1).

Other potential sources of unaccounted confounding are differences in other measures taken during the vaccination period versus previous periods and the increasing circulation of new variants with different infectiousness and fatality risk (Public Health England - G, 2021; European Centre for Disea, 2020; World Health Organization 2020; Toole et al., 2021). Non-pharmaceutical interventions changed continuously over time and across countries during the pandemic, and some of these measures may have resulted in shifting deaths to different age strata (Ioannidis, 2021b). E.g., relaxation of measures in 2021 may have been more prominent among non-elderly populations. This hypothesis is difficult to prove or disprove, but careful, serial seroprevalence studies (Bajema et al., 2021; Kshatri et al., 2021; Coyle et al., 2021; Stadlbauer et al., 2021; Bogogiannidou et al., 2020; Nisar et al., 2021) may help understand the potential change in population spread of the infection during 2021 versus 2020. Moreover, new variants did circulate widely in many countries during early 2021 (Konings et al., 2021). In particular, the Gamma variant became widely spread in South America (Taylor, 2021) and the Alpha variant became widespread in many high-income countries (Toole et al., 2021). There is some tentative evidence for increased infectivity (Boehm et al., 2021) and even potentially increased mortality risk (Challen et al., 2021). It is also possible that infection by some variants may be less likely to be prevented by prior infection (Imai et al., 2021). However, it is unclear whether these features would have affected the mortality footprint more prominently among younger populations.

Not all COVID-19 vaccines may be equally effective in curbing fatalities and effectiveness for decreasing mortality of some vaccines may vary across different age strata. Of note, a most prominent outlier in our analyses was Mexico, where the proportion of elderly COVID-19 deaths increased during 2021. Mexico has used 6 different vaccines, including Chinese vaccines (Sinovac/Sinopharm) that may have lower effectiveness than other licensed vaccines (Doroftei et al., 2021).

Finally, we did not assess for analysis the vaccination data and COVID-19 deaths after the first 5 months of 2021. We chose this, because differences in vaccination rates between different age groups diminished over time. Importantly, in most countries that prioritized vaccination of the elderly, most of the highly vulnerable individuals of younger age groups were likely to have been eventually vaccinated later in the year. If so, the age shift may be attenuated with further follow-up and this would have to be explored in future analyses in the fall and winter of 2021-2022. The emergence and dominance of the delta variant in the USA and Europe in mid-2021 adds another confounding factor in this regard. 


\subsection{Evolution of the pandemic and endemicity}

Acknowledging these caveats, our analyses show a substantial shift in the age distribution of COVID-19 deaths in countries that rapidly implemented vaccination predominantly among their elderly populations. Lower total fatalities and a shift towards the younger population probably represent favorable news of population-level vaccination effectiveness and a transition of the pandemic towards less threatening endemicity. Nevertheless, the demographic features of COVID-19 fatalities need to continue to be carefully scrutinized over time.

\section{Declaration of competing interest}

The authors declare that they have no known competing financial interests or personal relationships that could have appeared to influence the work reported in this paper.

\section{Acknowledgements}

We would like to thank Juan Fornos for kindly providing the Uruguay data he extracted from official sources.

\section{Appendix A. Supplementary data}

Supplementary data to this article can be found online at https://doi. org/10.1016/j.envres.2021.112342.

\section{Author Contributions}

All authors contributed to the study conception and design. Material preparation and data collection were performed by Roberta Pastorino, Leonardo Villani, Angelo Maria Pezzullo, Francesco Andrea Causio, Cathrine Axfors, Despina G. Contopoulos-Ioannidis. Roberta Pastorino and Angelo Maria Pezzullo performed the statistical analysis. The first draft of the manuscript was written by John P.A. Ioannidis, Despina G. Contopoulos-Ioannidis, Leonardo Villani, Roberta Pastorino, Angelo Maria Pezzullo and Stefania Boccia. John P.A. Ioannidis, Cathrine Axfors, Despina G. Contopoulos-Ioannidis and Stefania Boccia commented on the subsequent and latest version of the manuscript. John P. A. Ioannidis supervised the work. All authors have read and agreed to the published version of the manuscript.

\section{Declarations}

\section{Funding}

No funding was received for conducting this study.

\section{Ethics approval}

Not applicable.

\section{Patient and public involvement}

None.

\section{References}

Baden, L.R., El Sahly, H.M., Essink, B., Kotloff, K., Frey, S., Novak, R., et al., 2021 Efficacy and safety of the mRNA-1273 SARS-CoV-2 vaccine. N. Engl. J. Med. 384 (5), 403-416.

Bajema, K.L., Wiegand, R.E., Cuffe, K., Patel, S.V., Iachan, R., Lim, T., et al., 2021. Estimated SARS-CoV-2 seroprevalence in the US as of september 2020. JAMA Intern Med 181 (4), 450-460.

Benjamin, D.J., Berger, J.O., Johannesson, M., Nosek, B.A., Wagenmakers, E.J., Berk, R., et al., 2018. Redefine statistical significance. Nat Hum Behav 2 (1), 6-10. https:// doi.org/10.1038/s41562-017-0189-z. Available from:
Bernal, J.L., Andrews, N., Gower, C., Robertson, C., Stowe, J., Tessier, E., et al., 2021. Effectiveness of the Pfizer-BioNTech and Oxford-AstraZeneca vaccines on covid-19 related symptoms, hospital admissions, and mortality in older adults in England: test negative case-control study. BMJ 373.

Boehm, E., Kronig, I., Neher, R.A., Eckerle, I., Vetter, P., Kaiser, L., 2021. Novel SARSCoV-2 variants: the pandemics within the pandemic. Clin. Microbiol. Infect. S1198-743X (21), 262-267.

Bogogiannidou, Z., Vontas, A., Dadouli, K., Kyritsi, M.A., Soteriades, S., Nikoulis, D.J., et al., 2020. Repeated leftover serosurvey of SARS-CoV-2 IgG antibodies, Greece, march and April 2020. Euro Surveill. 25 (31), 1-6.

Centers for Disease Control and Prevention, 2020. COVID-19 Vaccination Program Interim Operational Guidance Jurisdiction Operations.

Challen, R., Brooks-Pollock, E., Read, J.M., Dyson, L., Tsaneva-Atanasova, K., Danon, L., 2021. Risk of mortality in patients infected with SARS-CoV-2 variant of concern 202012/1: Matched cohort study. BMJ 372, 1-10.

Connors, J., Bell, M.R., Marcy, J., Kutzler, M., Haddad, E.K., 2021. The impact of immuno-aging on SARS-CoV-2 vaccine development. GeroScience 43 (1), 31-51.

Coyle, P.V., Chemaitelly, H., Ben Hadj Kacem, M.A., Abdulla Al Molawi, N.H., El Kahlout, R.A., Gilliani, I., et al., 2021. SARS-CoV-2 seroprevalence in the urban population of Qatar: an analysis of antibody testing on a sample of 112,941 individuals. iScience 24 (6), 102646.

Dagan, N., Barda, N., Kepten, E., Miron, O., Perchik, S., Katz, M.A., et al., 2021. BNT162b2 mRNA Covid-19 vaccine in a nationwide mass vaccination setting. N. Engl. J. Med. 384 (15), 1412-1423.

Doroftei, B., Ciobica, A., Ilie, O.-D., Maftei, R., Ilea, C., 2021. Mini-Review discussing the reliability and efficiency of COVID-19 vaccines. Diagnostics 11 (4), 579.

European Centre for Disease Prevention and Control (ECDC), 2020. Rapid increase of a SARS-CoV-2 variant with multiple spike protein mutations observed in the Rapid increase of a SARS-CoV-2 variant with multiple spike protein mutations observed in the United Kingdom (December). Available from: http://covid19-country-overviews. ecdc.europa.eu/\#34_United_Kingdom.

European Centre for Disease Prevention and control, 2021. Overview of the Implementation of COVID-19 Vaccination Strategies and Vaccine Deployment Plans in the EU/EEA.

Fornos, J., 2021. Coviduy.com Github repository. Available from: https://github.com/b utaca/covid19uy.com.

Hussien, H., Nastasa, A., Apetrii, M., Nistor, I., Petrovic, M., Covic, A., 2021. Different aspects of frailty and COVID-19 : points to consider in the current pandemic and future ones. BMC Geriatr. 21 (1), 1-11.

Imai, M., Halfmann, P.J., Yamayoshi, S., Iwatsuki-horimoto, K., Chiba, S., 2021. Characterization of a new SARS-CoV-2 variant that emerged in Brazil. Proc. Natl. Acad. Sci. U. S. A. 118 (27), 1-9.

Institut national d'études démographiques (INED), 2020. The demography of COVID-19 deaths. Available from: https://dc-covid.site.ined.fr/en/.

Ioannidis, J.P., 2021 Jun. Over- and under-estimaiton of COVID-19 deaths. Eur. J. Epidemiol. 36 (6), 581-588.

Ioannidis, J.P.A., 2021b. Precision shielding for COVID-19: metrics of assessment and feasibility of deployment. BMJ Glob. Health 6 (1), 1-8.

Ioannidis, J.P.A., Axfors, C., Contopoulos-ioannidis, D.G., 2021. Second versus first wave of COVID-19 deaths : shifts in age distribution and in nursing home fatalities. Environ. Res. 195, 110856.

Koff, W.C., Schenkelberg, T., Williams, T., Baric, R.S., McDermott, Adrian, Cameron, C. M., et al., 2021. Development and deployment of COVID-19 vaccines forthose most vulnerable. Sci. Transl. Med. 13 (579), 1-15.

Konings, F., Perkins, M.D., Kuhn, J.H., Pallen, M.J., Alm, E.J., Archer, B.N., et al., 2021. SARS-CoV-2 Variants of Interest and Concern naming scheme conducive for global discourse. Nat. Microb. 6 (7), 821-823.

Kshatri, J.S., Bhattacharya, D., Praharaj, I., Mansingh, A., Parai, D., Kanungo, S., et al., 2021. Seroprevalence of SARS-CoV-2 in Bhubaneswar, India: findings from 3 rounds of community surveys. Epidemiol. Infect. 149, e139.

National Health Institute of Public Health (NIJZ), 2021. Dnevno Spremljanje Okužb S SARS-CoV-2 (COVID-19). Available from: https://www.nijz.si/sl/dnevno-spremljanj e-okuzb-s-sars-cov-2-covid-19.

National Institute of Population and Social Security Research - Japan, 2021. Data on COVID-19 - trend of COVID-19 Cases and deaths in Japan. Available from: http://www.ipss.go.jp/projects/j/Choju/covid19/index-en.asp.

Nisar, M.I., Ansari, N., Khalid, F., Amin, M., Shahbaz, H., Hotwani, A., Rehman, N., et al., 2021. Serial population-based serosurveys for COVID-19 in two neighbourhoods of Karachi, Pakistan. Int. J. Infect. Dis. 106, 176-182.

Polack, F.P., Thomas, S.J., Kitchin, N., Absalon, J., Gurtman, A., Lockhart, S., et al., 2020. Safety and efficacy of the BNT162b2 mRNA Covid-19 vaccine. N. Engl. J. Med. 383 (27), 2603-2615.

PopulationPyramid.net. Population Pyramids of the World from 1950 to 2100 [Internet]. Available from:: https://www.populationpyramid.net/.

Public Health England - Gov.uk, 2021. SARS-CoV-2 Variants of Concern and Variants under Investigation in England. Sage, pp. 1-50. April.

Riffe, T., Acosta, E., Acosta, E.J., Manuel Aburto, D., Alburez-Gutierrez, A., et al., 2021. Data Resource Profile: COVerAGE-DB: a global demographic database of COVID-19 cases and deaths. Int. J. Epidemiol. 50 (2), 390-390f.

Serwis Rzeczypospolitej Polskiej - Ministerstwo Zdrowia. Mapy Potrzeb Zdrowotnych -.

Stadlbauer, D., Tan, J., Jiang, K., Hernandez, M.M., Fabre, S., Amanat, F., et al., 2021. Repeated cross-sectional sero-monitoring of SARS-CoV-2 in New York City. Nature 590 (7844), 146-150.

Taylor, L., 2021. Covid-19: how the Brazil variant took hold of South America. BMJ 373, $1-3$. 
Toole, Á.O., Hill, V., Pybus, O.G., Watts, A., Bogoch II, Khan, K., et al., 2021. Tracking the International Spread of SARS-CoV-2 Lineages B. 1.1. 7 and B. 1.351/501Y-V2. Wellcome Open Research, p. 6.

University of Oxford - Blavatnik School of Government, 2020. Oxford COVID-19 government response tracker. Available from: https://www.bsg.ox.ac.uk/research /research-projects/covid-19-government-response-tracker.

Vasileiou, E., Simpson, C.R., Shi, T., Kerr, S., Agrawal, U., Akbari, A., et al., 2021. Interim findings from first-dose mass COVID-19 vaccination roll-out and COVID-19 hospital admissions in Scotland: a national prospective cohort study. Lancet 397 (10285), 1646-1657.
Voysey, M., Clemens, S.A.C., Madhi, S.A., Weckx, L.Y., Folegatti, P.M., Aley, P.K., et al., 2021. Safety and efficacy of the ChAdOx1 nCoV-19 vaccine (AZD1222) against SARS-CoV-2: an interim analysis of four randomised controlled trials in Brazil, South Africa, and the UK. Lancet 397 (10269), 99-111.

World Health Organization (WHO), 2020. Tracking SARS-CoV-2 variants. Available from: https://www.who.int/en/activities/tracking-SARS-CoV-2-variants/.

Worldometer. COVID-19 Coronavirus pandemic. Available from: https://www.wor ldometers.info/coronavirus/. 